\title{
Maroc 2007. Les élections législatives du 7 septembre
}

\author{
Jean-Luc Piermay
}

\section{OpenEdition}

\section{Journals}

\section{Édition électronique}

URL : http://journals.openedition.org/echogeo/2051

DOI : 10.4000/echogeo.2051

ISSN : 1963-1197

\section{Éditeur}

Pôle de recherche pour l'organisation et la diffusion de l'information géographique (CNRS UMR 8586)

\section{Référence électronique}

Jean-Luc Piermay, « Maroc 2007. Les élections législatives du 7 septembre », EchoGéo [En ligne], Sur le Vif, mis en ligne le 13 novembre 2007, consulté le 22 avril 2019. URL : http:// journals.openedition.org/echogeo/2051 ; DOI : 10.4000/echogeo.2051

Ce document a été généré automatiquement le 22 avril 2019

\section{(c) (i) (9)}

EchoGéo est mis à disposition selon les termes de la licence Creative Commons Attribution - Pas d'Utilisation Commerciale - Pas de Modification 4.0 International 


\title{
Maroc 2007. Les élections législatives du 7 septembre
}

\author{
Jean-Luc Piermay
}

1 Le 7 septembre 2007 ont eu lieu au Maroc les deuxièmes élections législatives du règne de Mohammed VI. Survenant alors que le royaume connaît des transformations économiques majeures, marquées par une volonté très forte d'arrimage à des dynamiques mondiales et par d'importants travaux d'infrastructures destinés à mettre le pays « à niveau », elles étaient espérées comme une étape dans la maturation politique du pays et craintes du fait d'une possible poussée islamiste. Organisées sous l'égide d'un ministre de l'Intérieur non partisan, elles ont été jugées très favorablement par les observateurs pour leur bon déroulement et leur transparence. Pour la première fois, par exemple, les résultats détaillés sont connus par préfectures et provinces, et par circonscriptions électorales. Mais l'analyse des résultats suppose d'abord un retour sur le contexte politique marocain et sur les protagonistes de ces élections.

2 Le Maroc est une monarchie, fondée sur la Constitution adoptée en 1996 par quasiconsensus politique. Les pouvoirs du Roi sont considérables, le plus important étant d'être Commandeur des croyants, ce qui lui assure la légitimité religieuse. Sur le plan plus strictement politique, il assure la fonction exécutive, nomme le Premier ministre, et les ministres sur proposition de celui-ci, préside le Conseil des ministres. Il peut même légiférer hors du Parlement. Ce dernier est composé de deux chambres, la Chambre des Représentants (députés) et la Chambre des conseillers. Élu pour cinq ans, il peut être dissous par le Roi. Le gouvernement est responsable à la fois devant le Roi et devant le Parlement. Dans les faits, le Roi s'appuie sur des conseillers très puissants et dispose de relais nombreux, par exemple à travers des ministères dits de souveraineté, dont la nomination échappe au Premier ministre. La place du Parlement, et même celle du gouvernement, est donc fortement relativisée.

3 Mais le pays est aussi engagé, notamment depuis l'avènement de Mohammed VI, dans une période de changements non seulement économiques mais politiques. Certes, un certain nombre de sujets restent hors du débat politique : le Roi, la religion, la patrie (en 
particulier le statut du Sahara, récupéré de l'Espagne). Les évolutions n'en sont pas moins notables. Des progrès dans la régularité des opérations électorales avaient déjà été observés en 2002. Un grand nombre de partis politiques sont en activité (33), même si tous les groupes islamistes ne sont pas été autorisés à se constituer en partis (ainsi, l'association Al Adl Wal Ihsane du cheikh Yassine). Les élections de 2007 devaient marquer une nouvelle étape de ce processus de démocratisation. Dans un souci de responsabilisation accrue des partis, le Roi avait notamment promis de choisir un Premier ministre partisan. Cela n'avait pas été le cas en 2002, puisqu'un Premier ministre technocrate, issu du patronat textile, Driss Jettou, avait été nommé. Celui-ci, en poste depuis lors, est d'ailleurs assez largement considéré comme un des meilleurs Premiers ministres que le Maroc ait jamais eu. Entouré d'une équipe aux compétences certes inégales mais parfois incontestables, il a obtenu des résultats notables dans les domaines de l'économie, des infrastructures, de l'habitat, du tourisme, certains autres comme l'agriculture, l'éducation, la santé, la justice restant en retrait.

4 Les conditions de formation du nouveau gouvernement, consécutif aux élections, éclairent l'enjeu électoral et montrent les limites de l'ouverture. Il est donc intéressant de les évoquer en préalable. À l'issue des élections, le Roi a tenu sa promesse, en nommant Premier ministre Abbas El Fassi, secrétaire général du parti de l'Istiqlal arrivé en tête du scrutin, malgré ses compétences discutées. Le nouveau Premier ministre a appelé 5 des 6 partis arrivés en tête à participer au gouvernement (à l'exception des islamistes du PJD). Incapable de gérer les prétentions des partis, il a dû recourir à l'arbitrage du Roi et de ses conseillers. Le résultat final est un gouvernement de 33 ministres, parmi lesquels « 17 n'ont pas d'appartenance politique claire »(Tel Quel, 294). Deux des partis (Mouvement Populaire et Union Constitutionnelle), s'estimant mal récompensés, sont passés dans l'opposition ; d'autres, pourtant au gouvernement, sortent fragilisés de cette épreuve. Le poids des ministres de souveraineté, des ministres proches du palais et des ministres technocrates parachutés dans un parti politique pour l'occasion (et pour deux d'entre eux, ayant changé de parti au bout de vingt-quatre heures), ne peut que susciter des doutes sur le nouveau rôle attribué aux partis. Le recto de la médaille est en revanche la compétence reconnue de la plupart des membres du gouvernement.

Le cas Fouad Ali El Himma illustre bien les particularités du système politique marocain. «Ami du Roi» (ils ont fait leurs études ensemble), ministre délégué à l'Intérieur sous le précédent gouvernement, il faisait figure malgré cette charge relativement secondaire de numéro deux du régime. Sa démission à la veille des élections pour se présenter à la députation, fonction considérablement plus modeste, a stupéfié, d'autant qu'il restait manifestement très proche du Roi. Cette proximité ainsi que son charisme lui ont valu la plus brillante élection du 7 septembre (avec l'ensemble de sa liste, cas unique), bien que ne bénéficiant d'aucune affiliation politique. Déjà, il joue un rôle essentiel au Parlement, constituant un groupe de 36 Parlementaires qui a débauché des députés dans d'autres partis, emportant la présidence de la Commission des Affaires étrangères (ce qui lui permet de continuer à suivre le dossier saharien). De plus, le gouvernement comporterait beaucoup de ses proches. Pour en finir, comme le nouveau gouvernement ne peut compter que sur une majorité relative, l'apport du groupe de Fouad Ali El Hima sera en permanence indispensable à celui-ci. Il semble qu'autour de lui, la recomposition de la scène partisane marocaine ait commencé, peut-être prélude à une nouvelle place des partis dans le jeu national.

6 Ces faits postérieurs aux élections montrent la fragilité des partis politiques marocains. Pour ces élections, six grands partis se présentaient, outre une foule de petites formations. Ils 
relèvent de plusieurs logiques. L'Istiqlal (PI) est le vieux parti fondé en 1944 dans la lutte pour l'indépendance. L'USFP (Union Socialiste des Forces Populaires), né en 1959, fut l'aile gauche du mouvement national auquel l'Istiqlal participait également. Après des années d'opposition sous le règne de Hassan II, elle accéda finalement au pouvoir à la fin de celui-ci, permettant la transition politique. D'autres organisations sont issues d'une volonté royale de peser sur le jeu politique ; ainsi, le Rassemblement National des Indépendants (RNI), constitué autour d'un ancien Premier ministre. Ces partis, de même que le Mouvement Populaire (MP) et l'Union Constitutionnelle (UC) sont surtout devenus des machines à accéder au pouvoir, aux programmes incertains. Le Parti de la Justice et du Développement (PJD) tranche de manière évidente du fait de sa participation constante à l'opposition. Ce parti islamique est né en 1996 d'une association, comme les trois autres (petites) formations professant un islam politique. Utilisant un référentiel religieux, il doit composer entre de fortes aspirations de sa base en matière de justice sociale et un souci de respectabilité qui l'a fait faire allégeance au Roi et qui le fait apparaitre comme un parti modéré. Son programme cible en priorité la moralisation de l'administration, la justice et l'école. Car, grande nouveauté de ces élections, les partis ont publié des programmes de gouvernement, destinés toutefois à s'effacer dans l'exercice du pouvoir pour deux raisons structurelles. La première est que le mode de scrutin adopté, proportionnel au plus fort reste par circonscriptions, nécessite la mise en place de larges coalitions pour former les gouvernements. La seconde, encore plus forte, est que les gouvernements ont surtout à appliquer le programme du Roi. La conséquence principale de cette faiblesse des partis est le large discrédit qui les touche dans la population. Le Parlement lui-même est considéré comme peu utile. En revanche, le Roi est adulé.

7 Les résultats des élections du 7 septembre 2007 reflètent évidemment ce contexte. Le faible taux de participation ( $37 \%$, contre $52 \%$ en 2002), doublé du taux élevé de bulletins nuls (19 \%) a fortement choqué la classe politique. Ainsi, $70 \%$ des électeurs disposant de leur carte n'ont pas participé au vote. Ce sont les villes qui ont le moins exprimé leur vote : $72 \%$ d'abstentions et de bulletins nuls à Rabat, 77 \% à Marrakech et Salé, 80 \% à Fès et Meknès, $81 \%$ à Casablanca (et beaucoup moins dans certains quartiers populaires : $86 \%$ dans la Préfecture d'Al Fida Mers Sultan), 85 \% à Tanger. En revanche, ce sont les Provinces sahariennes qui ont le plus exprimé leur vote (46\%).

Ces données relativisent fortement les performances des partis. En nombre de voix, le PJD arrive en tête (10,9 \% des voix), loin derrière le raz-de-marée craint par certains, suivi par l'Istiqlal (10,7 \%), le RNI (9,7 \%), le MP (9,2 \%), l'USFP (8,8 \%), l'UC (7,2\%). Ces scores reflètent une grande stabilité du corps électoral, à l'exception de la montée des islamistes modérés (qui présentaient pour la première fois des candidats dans toutes les circonscriptions sauf une) et d'un déclin de l'USFP. Toutefois, par le jeu des découpages électoraux, l'Istiqlal arrive en tête en sièges (46 sur 295), suivi du PJD (40;41 avec un petit parti islamique), du MP (36), du RNI (34), de l'USFP (33) de l'UC (27).

9 La répartition géographique des votes apparaît à la fois contrastée et complexe. Aucun parti n'est implanté de manière régulière dans l'espace, et de loin. Ainsi, le score de l'Istiqlal par province va de 1 à $44 \%$, celui du PJD de 1,7 à $36 \%$, etc. En même temps, les répartitions suivent rarement des logiques simples. Le seul parti dont la répartition ne ressemble pas à une mosaïque est le PJD. Son implantation est fondamentalement urbaine et surtout concentrée le long de l'axe atlantique, de Tanger (31,7 \%) à Casablanca (19,8\% ), en passant par Tetouan (20,9\%), Larache (36,1\%), Salé (24,7 \%), Rabat (25,9\%). Hors de cet axe, on ne trouve guère que Fès $(30,4 \%)$, Oujda $(20,8 \%)$, Agadir $(25,9 \%)$, Meknès $(13,5 \%)$, toutes des villes importantes. Pour le reste, Ail y a quelques tonalités 
géographiques fortes. Ainsi, les Provinces sahariennes sont le seul lieu de forte polarisation, au profit des partis marocains historiques (37,8 \% pour l'Istiqlal, $25,4 \%$ pour l'USFP, 14,6 \% pour le MP) : sans doute s'agit-il d'un réflexe légitimiste dans une région dont le statut reste internationalement incertain. À cette explication s'en ajoute une autre, valable pour l'ensemble du pays : les votes, notamment en milieu rural, font une large place aux allégeances traditionnelles, celles des notables et des tribus. Cette situation ne favorise évidemment ni les positionnements politiques clairs des partis, ni leur lisibilité à l'échelle nationale, indispensables au moment de faire les choix dont le pays a besoin. C'est aussi pour cela que le PJD, qui se fonde plutôt sur le sentiment de marginalisation d'une grande partie de la population, ne pénètre pas (encore ?) les campagnes et pas plus l'espace suburbain de Casablanca.

Une question se pose enfin par rapport aux grands projets et chantiers que connait actuellement le pays : comment ont réagi les électeurs des régions concernées ? La ville de Tanger, marquée par de nombreux chantiers et voisine du grand port dont l'activité démarre sur la Méditerranée, atteint presque le record des votes islamistes. En revanche, la préfecture très rurale coïncidant avec le projet du port Tanger Méditerranée reste dominée par les paris de notables. Quant à Rabat-Salé, entre lesquelles l'aménagement de la vallée du Bou Regreg tend à conforter l'image de la capitale mais aussi à effectuer la soudure entre ces deux villes très dissemblables, l'une administrative et plutôt aisée, l'autre industrielle et pauvre, les résultats électoraux sont de part et d'autre étonnamment semblables, avec une forte poussée islamiste. Sans qu'il soit possible de dire si les grands chantiers ont renforcé le sentiment de l'exclusion chez certaines fractions de la population, les résultats des élections montrent en tout cas l'urgence qu'il y a de répondre à la question angoissante de l'emploi.

11 Si le Maroc n'a pas connu la poussée islamiste crainte par les opinions publiques occidentales et par une intelligentsia marocaine, la situation politique du pays n'en est pas moins délicate. La croissance économique bénéficie d'un pouvoir de décision fort et réactif autour d'un Roi qui ne se désintéresse nullement des questions sociales (INDH, Initiative Nationale pour le Développement Humain). Se pose toutefois un grave problème de réappropriation des changements par les populations. Le sentiment des injustices, de la corruption, de la croissance des inégalités -classique en période de croissance économique- aggravent les tensions sociales. Actuellement, les partis politiques sont incapables de jouer un rôle de médiation. S'il est de bon ton de fustiger les hommes politiques, il faut aussi reconnaître que la place laissée aux partis par le pouvoir royal, à travers la répartition des pouvoirs, les pratiques de pouvoir et les modes de scrutin, les condamne à l'impuissance et au discrédit. Dans cette brèche peuvent s'insérer des courants de pensée extérieurs aux partis, par exemple des idéologies islamistes non désireuses de composer avec le système. Celles-ci attirent certainement de nombreux sympathisants, mais sont encore trop peu organisées pour constituer une forte menace. Mais le changement peut aussi venir du Roi. Le problème est ici que la réforme de la vie politique et la consolidation des partis sont nécessaires l'une à l'autre et que le changement est tout autant indispensable que périlleux, pour un pays en pleine transformation. C'est peut-être là que se place la stratégie qui semble actuellement se dessiner autour de l'homme politique atypique qu'est Fouad Ali El Himma. 


\section{RÉSUMÉS}

$\mathrm{Au}$ Maroc, ont eu lieu les deuxièmes élections législatives du règne de Mohamed VI. Elles n'ont pas été marquées par la poussée islamiste attendue, et n'ont guère modifié l'équilibre entre les partis politiques. Toutefois, la faiblesse de la participation électorale laisse penser que la réappropriation par la population des grands changements en cours n'est pas acquise. Plus que jamais et au-delà de sa volonté de changement en ce domaine, le Roi est le maître de la vie politique.

In Morocco, September 7th 2007 legislative elections. In Morocco, the second legislative elections of Mohamed VI's reign took place. They were not marked by waited Islamic increase, and hardly changed balance between the political parties. Nevertheless, the weakness of electoral participation lets think that the re appropriation of the big ongoing changes by people is not acquired. More than ever and beyond his will of change, the king remains the master of political life.

\section{INDEX}

Mots-clés : Maroc, élections, changement, pouvoirs

Keywords : Morocco, change, authorities

\section{AUTEUR}

\section{JEAN-LUC PIERMAY}

Jean-Luc Piermay (jlp@equinoxe.u-strasbg.fr) est professeur à l'Université Louis Pasteur de Strasbourg. Actuellement, il est en détachement à l'UR 023 de l'IRD. Il a récemment publié avec Cheik Sarr La ville sénégalaise, une invention aux frontières du monde aux éditions Karthala, et participé à l'ouvrage dirigé par René Kahn Régulations temporelles et territoires urbains. Habiter l'espace et le temps d'une ville, publié aux Editions L'Harmattan. 